\title{
ПЕРСПЕКТИВНЫЕ МЕТОДЫ МОНИТОРИНГА ПОСЛЕДСТВИЙ РАЗЛИВОВ НЕФТЕПРОДУКТОВ ПО ДАННЫМ ДИСТАНЦИОННОГО ЗОНДИРОВАНИЯ ЗЕМЛИ
}

\author{
Антонина Сергеевна Гордиенко \\ Сибирский государственный университет геосистем и технологий, 630108, Россия, г. Ново- \\ сибирск, ул. Плахотного, 10, кандидат технических наук, доцент кафедры фотограмметрии \\ и дистанционного зондирования, тел. (383)361-08-66, e-mail: a.s.gordienko@sgugit.ru
}

В статье обоснована актуальность исследования и разработки методов применения данных дистанционного зондирования Земли при мониторинге последствий разливов нефтепродуктов. Описываются основные методы распознавания загрязнений нефтью водной поверхности и суши. Приводятся их достоинства и недостатки. Обсуждаются результаты выполненного обзора. Сделан вывод о необходимости определить оптимальное соотношение пространственного и спектрального разрешения снимков для установления связи между загрязнением нефтью и угнетением растительности. Предложены направления дальнейших исследований по разработке методов мониторинга последствий разливов нефти по данным дистанционного зондирования Земли с целью повышения их достоверности за счет комплексного подхода и адаптации к различным природно-территориальным комплексам.

Ключевые слова: последствия разливов нефтепродуктов, данные дистанционного зондирования, мониторинг, негативное воздействие, точность распознавания, нефтяные пленки, радиолокационные снимки, многоспектральные снимки

\section{PROSPECTIVE METHODS OF MONITORING RESULTS OF OIL SPILLS ACCORDING TO REMOTE SENSING DATA OF EARTH}

\section{Antonina S. Gordienko}

Siberian State University of Geosystems and Technologies, 10, Plakhotnogo St., Novosibirsk, 630108, Russia, Ph. D., Associate Professor, Department of Photogrammetry and Remote Sensing, phone: (383)361-08-66, e-mail: a.s.gordienko@sgugit.ru

The article presented the relevance of research and development of methods of monitoring results of oil spills by remote sensing data. The article describe the main methods of recognition of oil water surface and land pollution. The article provides the description and outlines disadvantages and advantages of all of methods. The conclusion is made about necessary to determine the spatial and spectral resolution of images to define a relationship between oil pollution and oppression of vegetation. The conclusion is made about the development of methods of monitoring results of oil spills by remote sensing data for increase their reliability and adaptation to various naturalterritorial complexes.

Keywords: results of oil spills, remote sensing data, monitoring, negative impact, recognition accuracy, oil pollution, radar images, multispectral images

В настоящее время, аварии на нефтепроводах, при добыче, транспортировке и хранении нефтепродуктов далеко не редкое явление. Нефть оказывает негативное влияние на окружающую среду и процесс восстановления природных ресурсов может затянуться на долгие годы. Кроме того, площадь поражения 
может быть достаточно обширной и охватывать труднодоступные районы. Оптимальным методом мониторинга в данном случае является использование данных дистанционного зондирования Земли (ДДЗ3).

Таким образом, цель данной статьи - анализ методов мониторинга последствий разливов нефтепродуктов по данным дистанционного зондирования земли. Для достижения поставленной цели необходимо решить следующие задачи:

- рассмотреть отечественный и зарубежный опыт распознавания разливов нефтепродуктов по ДДЗ3;

- проанализировать возможность распознавания результатов негативного воздействия нефти на окружающую среду;

- сформулировать соответствующие выводы.

Для распознавания разливов нефтепродуктов применяются данные получаемые как активными, так и пассивными съемочными системами. Кроме того, можно разделить все методы на те, что направлены на анализ водной поверхности и на распознавание загрязнений на суше.

Радиолокационные данные, как правило, используются для распознавания нефтяных пятен на водной поверхности (рис. 1). Наиболее подходящим для этих целей является X-диапазон [1-7]. Кроме того, как известно, радиолокационные данные позволяют получать информацию как в дневное время, так и в ночное, а также плохую погоду. Что дает им преимущество по сравнению с данными в видимом диапазоне спектра.

Точность распознавания разливов нефти по радиолокационным изображениям зависит от ряда факторов: скорость ветра, тип и количество нефтепродуктов, поступивших в воду.

Часто радиолокационные изображения нефтяных пленок на водной поверхности можно спутать с органическими пленками, некоторыми типами льдин, участками, затененными сушей и др. [8-12] При скорости ветра, превышающей 9-10 м/с, нефтяные пленки становятся неотличимы от водной поверхности. [13]

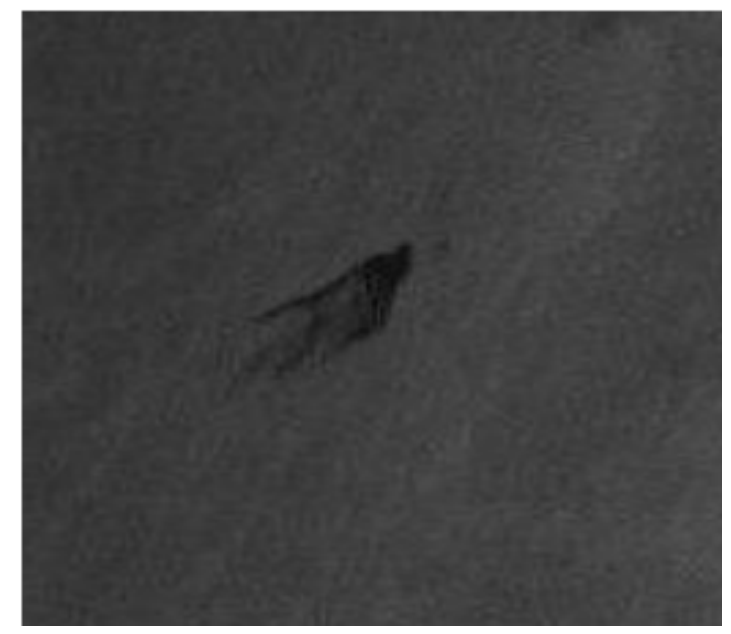

Рис. 1. Фрагмент радиолокационного снимка с изображением нефтяного пятна на водной поверхности 
Снимки, полученные в видимом диапазоне спектра, не позволяют однозначно распознавать разливы нефти этому мешает наличие солнечных бликов. Кроме того, затрудняет распознавание облачность и невозможность использования снимков, полученных в темное время суток [14-16]. Фрагмент снимка Sentinel-2 с изображением разлива нефти на реке представлен на рис. 2.

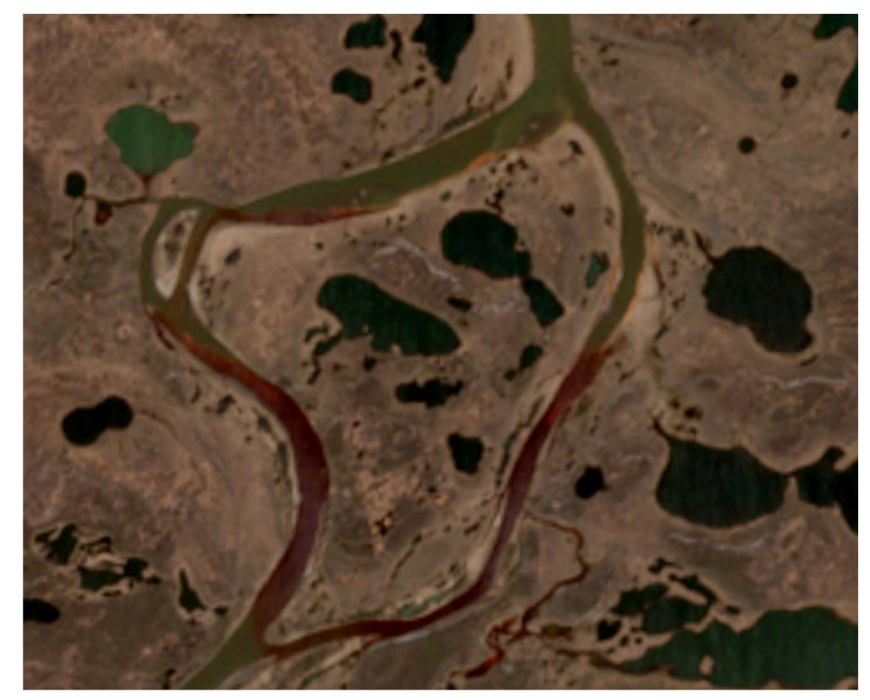

Рис. 2. Фрагмент снимка Sentinel-2 с изображением разлива нефти на реке

Использование оптических изображений совместно с радиолокационными повышает достоверность распознавания нефтяных пятен на водной поверхности. [13]. Так как контраст между нефтью и чистой водой определяется их оптическими характеристиками.

Изображения нефти в инфракрасном диапазоне спектра можно спутать с водорослями и другими органическими веществами [17-19].

Ближний инфракрасный диапазон спектра может применяться для определения остаточной заболоченности нефтью, но метод требует дополнительных исследований [20-21].

Ультрафиолетовое излучение, также однозначно не определяет нефтяные разливы. Этому мешают солнечные блики и биологический материал [22].

Микроволновый сигнал изменяется в зависимости от толщины масла, поэтому для измерения толщины нефтяного пятна можно использовать радиометрию. Однако существуют помехи, кроме того микроволновый радиометр имеет низкое пространственное разрешение (обычно десятки метров). В настоящее время акцент в исследованиях делается на использовании нескольких длин волн микроволнового излучения для измерения толщины пятна [23].

Метод выявления нефтяных пленок описанный в [24], позволяет обнаруживать пленки по гиперспектральным данным на основе системы спектральных признаков. Но он позволяет классифицировать пленки только на два класса: мощные и тонкие, и применим только для обнаружения загрязнений на водной поверхности [24-25]. 
Распознавание разливов нефти на суше более сложный процесс. Для этого необходимы многоспектральные данные с высоким пространственным разрешением [26]. Косвенным признаком наличия нефти в данном случае будет состояние растительности, которое связано с воздействием на нее нефти [27].

Покрытие листьев нефтью затрудняет газообмен между ними и атмосферой. Что в последствии приведет к гибели растений. Косвенное воздействие проявляется в том, что наличие нефтепродуктов в почве, влияет на круговорот питательных веществ, который приведет к ухудшению состояния флоры. Так как различные типы угодий имеют разный уровень восприимчивости к воздействию нефти, восстановление природно-территориальных комплексов может занять от нескольких месяцев до нескольких лет [28-30].

Как правило, разливы нефти и загрязнение окружающей среды (почва и растительность) определяют по значениям индексов, которые характеризуют ее состояние. Но для получения точных данных необходимо учитывать типы ландшафтов, сезон, погодные условия и др. Размер нефтяных луж и площадь поражения, определяемые по снимкам, зависят от разрешающей способности съемочной системы. [31-35]

Определение толщины нефтяных пятен является важным моментом для оценки эффективности контрмер и в целях судебного преследования. Существует несколько методов оценки толщины пятна в диапазоне 0,1-5 мкм, но они не подходят в большинстве случаев. Часто требуется измерение толщины менее 0,5 мм [36].

Были попытки соотнести толщину масла с его внешним видом и цветом. Дальнейшие исследования показывают, что этот метод ограничен блеском и радужными пятнами. Физически обоснованные толщины около 0,7-2,5 мм выглядят как цвета радуги.

Предполагалось, что по изображениям, полученным в инфракрасном диапазоне спектра можно определять толщину нефтяной пленки, но обширные испытания нескольких исследователей показали, - яркость инфракрасного излучения не зависит от толщины пятна [36-37].

\section{Заключение}

Выбор подходящих данных дистанционного зондирования для обнаружения и мониторинга последствий разливов при добыче, транспортировке и хранении нефти требует тщательного рассмотрения затрат и возможности достижения целей, поскольку ни один датчик не отвечает всем требованиям, необходимым для решения такой задачи.

То есть, необходимо определить оптимальное соотношение пространственного и спектрального разрешения снимков для установления связи между загрязнением нефтью и угнетением растений.

Дальнейшие исследования потребуют рассмотрения таких факторов, как особенности распознавания негативного влияния нефтепродуктов на окружающую среду в различных климатических поясах, реакция различных видов рас- 
тений на разлив нефти, её тип, характеристики спутникового датчика, (спектральное и пространственное разрешение), временной интервал между разливом и датой сьемки и объем нефти, который может оказать влияние.

Таким образом, необходимо совершенствование методов определения типов и масштабов загрязнений нефтепродуктами с целью получения количественных характеристик и адаптации к различным природно-территориальным системам.

Работа выполнена в рамках государственного задания Минобрнауки России (тема «Разработка теории и технологических решений контроля состояния защитных сооружений при перекачке нефтепродуктов методами активного дистаниионного зондирования», № 0807-2020-0002).

\section{БИБЛИОГРАФИЧЕСКИЙ СПИСОК}

1. Marzialetti, P., Laneve, G. Oil spill monitoring on water surfaces by radar L, C and X band SAR imagery: A comparison of relevant characteristics. In Proceedings of the 2015 IEEE International Geoscience and Remote Sensing Symposium (IGARSS), Beijing, China, 10-15 July 2016. C. $7715-7717$.

2. Liu, P., Zhao, C., Li, X., He, M., Pichel, W. Identification of ocean oil spills in SAR imagery based on fuzzy logic algorithm. Int. J. Remote Sens. 2010. - 31. - C. $4819-4833$.

3. Minchew, B., Jones, C.E., Holt, B. Polarimetric analysis of backscatter from the Deepwater Horizon oil spill using L-band synthetic aperture radar. IEEE Trans. Geosci. Remote Sens. 2012. 50. - C. 3812-3830.

4. Nunziata, F., Buono, A., Migliaccio, M. A new look at the old sea oil slick observation problem: Opportunities and pitfalls of SAR polarimetry. In Proceedings of the 2015 IEEE International Geoscience and Remote Sensing Symposium (IGARSS), Beijing, China, 10-15 July 2016. C. 4027-4030.

5. Collins, M.J., Denbina, M., Minchew, B., Jones, C.E., Holt, B. On the Use of Simulated Airborne Compact Polarimetric SAR for Characterizing Oil-Water Mixing of the Deepwater Horizon Oil Spill. IEEE J. Sel. Top. Appl. Earth Obs. Remote Sens. 2015. - 8. - C. 1062-1077.

6. Guo, H., Wu, D., An, J. Discrimination of Oil Slicks and Lookalikes in Polarimetric SAR Images using CNN. Sensors 2017. - 17. - $1837 \mathrm{c}$.

7. Chen, G., Li, Y., Sun, G., Zhang, Y. Application of Deep Networks to Oil Spill Detection Using Polarimetric SAR Images. Appl. Sci. 2017. - 7. - 968 c.

8. Oil pollution in the North Sea: The Handbook of Environmental Chemistry (2016) / ed. Carpenter A. Springer, 2016. - V. 41. - 312 c.

9. Oil pollution in the Mediterranean Sea. Pt. 1. The international context: The Handbook of Environmental Chemistry (2017a) / eds. Carpenter A., Kostianoy A.G. Springer, 2017.

10. Oil pollution in the Mediterranean Sea. Pt. 2. National case studies: The Handbook of Environmental Chemistry. (2017b) / eds. Carpenter A., Kostianoy A.G. Springer, 2017.

11. Kostianoy A.G., Lavrova O.Yu., Mityagina M. I., Solovyov D.M. (2014c) Satellite monitoring of the Nord Stream gas pipeline construction in the Gulf of Finland // Oil Pollution in the Baltic Sea. V. 27 / eds. A.G. Kostianoy, O.Yu. Lavrova. Springer, 2014. - C. 221-248.

12. Kostianoy A.G., Ginzburg A. I., Kopelevich O.V., Kudryavtsev V.N., Lavrova O.Yu., Lebedev S.A., Mitnik L.M., Mityagina M. I., Smirnov V.G., Stanichny S.V., Troitskaya Yu. I. (2017) Ocean Remote Sensing in Russia // Comprehensive Remote Sensing. 2017. - V. 8. Ocean Remote Sensing. Elsevier, 2017.

13. Лаврова О.Ю., Митягина М.И., Костяной А.Г. Спутниковые методы выявления и мониторинга зон экологического риска морских акваторий.- М.: ИКИ РАН, 2016.— 334 с. 
14. Leifer, I., Lehr, B., Simecek-Beatty, D., Bradley, E., Clark, R., Dennison, P., Hu, Y.; Matheson, S., Jones, C., Holt, B., et al. State of the art satellite and airborne oil spill remote sensing: Application to the BP DeepWater Horizon oil spill. Remote Sens. Environ. 2012. - 124. - C. 185-209.

15. Iler, A.L., Hamilton, P.D. Detecting oil on water using polarimetric imaging. Proc. SPIE Int. Soc. Opt. Eng. 2015. - 9459 c.

16. Shen, H.-Y., Zhou, P.-C., Feng, S.-R. Research on multi-angle near infrared spectralpolarimetric characteristic for polluted water by spilled oil. Proc. SPIE Int. Soc. Opt. Eng. 2011. $8193 \mathrm{c}$.

17. Chenault, D.B., Vaden, J.P., Mitchell, D.A., Demicco, E.D. Infrared polarimetric sensing of oil on water. Proc. SPIE Int. Soc. Opt. Eng. 2016. - 9999 c.

18. Pinel, N., Monnier, G., Sergievskaya, I., Bourlier, C. Simulation of infrared emissivity and reflectivity of oil films on sea surfaces. Proc. SPIE Int. Soc. Opt. Eng. 2015. - 9638 c.

19. Shih, W.-C., Andrews, A.B. Infrared contrast of crude-oil-covered water surfaces. Opt. Lett. 2008. - 33. - C. $3019-3021$.

20. Kokaly, R.F., Couvillion, B.R., Holloway, J.M., Roberts, D.A., Ustin, S.L., Peterson, S.H., Khanna, S., Piazza, S.C. Spectroscopic remote sensing of the distribution and persistence of oil from the Deepwater Horizon spill in Barataria Bay marshes. Remote Sens. Environ. 2013. - 129. - C. 210-230.

21. Sicot, G., Lennon, M., Miegebielle, V., Dubucq, D. Analysis of the reflectance spectra of oil emulsion spilled on the sea surface. Proc. SPIE Int. Soc. Opt. Eng. 2014. - 9240 c.

22. Yin, D., Huang, X., Qian, W., Huang, X., Li, Y., Feng, Q. Airborne validation of a newstyle ultraviolet push-broom camera for ocean oil spill pollution surveillance. Proc. SPIE Int. Soc. Opt. Eng. 2010. - 7825 c.

23. Fingas, M., Brown, C. Review of oil spill remote sensing. Mar. Pollut. Bull. 2018. - 18. C. 9-23.

24. Жуков, Д.В. Спектральные признаки для идентификации типовых загрязнений акваторий морей по данным авиационной и космической съемки / Д.В. Жуков // Оптика атмосферы и океана. - 2016. - Т. 29. №07. - С. 560-565.

25. Патент RU 2440566. Способ дистанционного обнаружения нефтяных загрязнений на поверхности воды. Дата действия патента 27.07.10. МПК G01N 21/55.

26. Khanna, S., Santos, M.J., Ustin, D.S.L., Koltunov, A., Kokaly, R.F., Roberts, D.A. Detection of salt marsh vegetation stress after the Deepwater Horizon BP oil spill along the shoreline of gulf of Mexico using AVIRIS data. PLoS ONE 2013. - 8. e78989.

27. Noomen, M., Smith, K. L., Colls, J. J., Stevens, M. D., Skidmore, A. K., \& van der Meer, F. D. (2008). Hyperspectral indices for detecting changes in canopy reflectance as a result of underground natural gas leakage. International journal of remote sensing, 29(20). - C. 5987-6008.

28. Hester, M.W., Mendelssohn, I.A. Long-term recovery of a Louisiana brackish marsh plant community from oil-spill impact: Vegetation response and mitigating effects of marsh surface elevation. Mar. Environ. Res. 2000. - 49. - C. 233-254.

29. Khanna, S., Santos, M., Koltunov, A., Shapiro, K., Lay, M., Ustin, S. Marsh Loss Due to Cumulative Impacts of Hurricane Isaac and the Deepwater Horizon Oil Spill in Louisiana. Remote Sens. 2017. - 9. - 169 c.

30. Токарева О.С., Климентьев, Д.С. Оценка последствий нефтяных разливов на основе данных дистанционного зондирования Земли // Интерэкспо Гео-Сибирь. - Новосибирск: СГГА, 2010. Т. 4. - С. 130-133.

31. Алексеева М.Н., Ященко И.Г. Использование космических снимков и геоданных для оценки экологических рисков при разливах нефти // Интерэкспо Гео-Сибирь. - Новосибирск: СГГА, 2013. Т. 7. - С. 55-60.

32. Ефимова М.В., Стрих Н.И., Курбанов В.Ш.. Воздействие нефтегазового комплекса на экосистемы Ханты-Мансийского автономного округа-Югры // Региональные геосистемы, 2011. - T. 14, .3-1 (98). - C. 110-114. 
33. Ященко И.Г., Перемитина Т.О. Мониторинг экологического состояния нефтедобывающих территорий Западной Сибири с применением данных дистанционного зондирования // Интерэкспо Гео-Сибирь. - Новосибирск: СГГА, 2015. Т. 4. - С. 89-93.

34. Разакова М.Г. Выявление и картирование нефтяных загрязнений почв по данным дистанционного зондирования // Проблемы информатики, 2017. - 4 (37). - С. $4-15$.

35. Кусков А.П. Применение данных дистанционного зондирования земли при инвентаризации нефтезагрязненных земель // Наука, техника и образование, 2017. - 7 (37). - С. 111-115.

36. Fingas, M.F. How to measure slick thickness (or not). In Proceedings of the 35 th AMOP Technical Seminar on Environmental Contamination and Response, Vancouver, BC, Canada, 5-7 June 2012. - C. 617-652.

37. Shih, W.-C., Andrews, A.B. Infrared contrast of crude-oil-covered water surfaces. Opt. Lett. 2008. - 33. - C. 3019-3021.

(C) A. С. Гордиенко, 2021 